\title{
Intrinsic low-frequency variability of the Gulf Stream
}

\author{
G. Quattrocchi ${ }^{1}$, S. Pierini ${ }^{1}$, and H. A. Dijkstra ${ }^{2}$ \\ ${ }^{1}$ Department of Environmental Sciences, University of Naples "Parthenope", Naples, Italy \\ ${ }^{2}$ Institute for Marine and Atmospheric research Utrecht, Department of Physics and Astronomy, Utrecht University, Utrecht, \\ The Netherlands
}

Correspondence to: G. Quattrocchi (giovanni.quattrocchi@uniparthenope.it)

Received: 10 August 2011 - Revised: 15 February 2012 - Accepted: 18 February 2012 - Published: 5 March 2012

\begin{abstract}
In this paper a process study aimed at analyzing the low-frequency variability of intrinsically oceanic origin of the Gulf Stream (GS) and GS extension (GSE) is presented. An eddy-permitting reduced-gravity nonlinear shallow water model is implemented in an idealized North Atlantic Ocean, with schematic boundaries including the essential geometric features of the coastline and a realistic zonal basin width at all latitudes. The forcing is provided by a time-independent climatological surface wind stress obtained from 41 years of monthly ECMWF fields. The model response yields strong intrinsic low-frequency fluctuations on the interannual to decadal time scales. The modelled timeaveraged GS/GSE flows are found to exhibit several features that can also be deduced from satellite altimeter data, such as the Florida Current seaward deflection, the GS separation at Cape Hatteras, and the overall structure of the GSE. The intrinsic low-frequency variability yields two preferred states of the GSE differing in latitudinal location that also have their counterpart in the altimeter data. A preliminary analysis of the variability in terms of dynamical systems theory is carried out by using the lateral eddy viscosity as the control parameter. A complex transition sequence from a steady state to irregular low-frequency variability emerges, in which Hopf and global bifurcations can be identified.
\end{abstract}

\section{Introduction}

Along the western sides of the main world ocean basins western boundary currents (WBCs) flow as intense and narrow streams, transporting huge volumes of warm and salty water poleward. The strongest currents are located in the Northern Hemisphere: the Gulf Stream (GS) in the North Atlantic Ocean and the Kuroshio in the North Pacific Ocean. The GS and Kuroshio and their extension jets (GSE and KE, respectively) profoundly affect the global climate system. The high eddy kinetic energy level, the strong large-scale interannual to decadal changes and corresponding air-sea interaction processes present in the KE region are known to affect considerably the climate of the North Pacific area (e.g. Qiu, 2000, 2002; Kelly et al., 2010). The GSE interannual variability is mainly associated with a meridional shift of the jet (e.g. Lee and Cornillon, 1995; Frankignoul et al., 2001; Zhang and Vallis, 2006; Sasaki and Schneider, 2011a), and can drive energetic synoptic atmospheric fluctuations of climatic relevance (e.g. Joyce et al., 2009; Feliks et al., 2011).

In situ investigations, three decades of satellite missions and many model studies have provided clear evidence of the large variability of such WBCs, particularly after their separation from the coast (for recent overviews, see Kelly et al., 2010; Rossby et al., 2010; Sasaki and Schneider, 2011b). One of the most striking differences between the GS/GSE and Kuroshio/KE is the low-frequency variability of two characteristic indices, the path latitude and the surface transport (see Fig. 12 in Kelly et al., 2010). While the KE clearly shows large amplitude variations on a decadal time scale, interannual variability dominates in the GSE. In fact, while decadal bimodality of the KE path is well established from observations (Taft, 1972; Qiu, 2002; Qiu and Chen, 2010), in the GS Extension (GSE) such a strong bimodality is absent, although significantly fluctuating meandering patterns are present. As for the GS, following the southeast coastline of the United States it yields a weak seaward deflection off South Carolina, as revealed by satellite observations. Bane and Dewar (1988) suggested that such a deflection has a bimodal character, but with transitions occurring on a relatively fast (inter-monthly) time scale. By analyzing yearly averages of the cold wall of the GS, Auer (1987) showed that a weak interannual variability is also present in the same region (see Fig. 4 in Auer, 1987).

As far as the cause of the observed variability is concerned, low-frequency fluctuations in the wind forcing are known to play a major role in GSE changes. For instance, shifts of the GSE axis lag atmospheric fluctuations associated with 
the North Atlantic Oscillation by few years (Frankignoul et al., 2001; de Coëtlogon et al., 2006; Sasaki and Schneider, 2011a). On the other hand it is well known that highly nonlinear WBC extensions, such as the GSE, can, under certain conditions exhibit strong low-frequency variability generated exclusively by intrinsic oceanic mechanisms (Dijkstra, 2005; Dijkstra and Ghil, 2005). It is therefore of great theoretical interest to investigate how nonlinear internal ocean dynamics contributes to the GSE variability.

Model studies of the intrinsic low-frequency variability in the wind-driven ocean circulation in idealized rectangular basins (e.g. Jiang et al., 1995; McCalpin and Haidvogel, 1996; Dijkstra and Katsman, 1997) and in configurations with realistic continental geometry and steady wind stress (e.g. Dijkstra and Molemaker, 1999; Schmeits and Dijkstra, 2001; Pierini, 2006) suggest that multiple equilibria and intrinsic low-frequency variability due to nonlinear mechanisms all internal to the ocean system may be present in both the North Atlantic and North Pacific WBC extensions. More recent work has suggested that energetic intrinsic relaxation oscillations contributing to the interannual and decadal-scale variability in WBC extensions (and in the KE in particular) may arise from a global bifurcation in phase space (Simonnet et al., 2005; Pierini, 2006; Pierini et al., 2009). In simulations based on a reduced-gravity shallow water model with schematic coastline, the behaviour of the KE path length and upstream path position (Pierini, 2006; Pierini et al., 2009; Pierini and Dijkstra, 2009) is found to be in quantitative agreement with observations.

As the GS/GSE system is dynamically analogous to the Kuroshio/KE system in terms of multiple equilibria and intrinsic variability, an interesting issue is what variability is found when the shallow water model of Pierini et al. (2009) is applied to the North Atlantic region. Differences with the North Pacific in this context will include the schematic coastline, the representation of the upper-layer stratification and the climatological wind stress. Will decadal-scale transitions between large-scale GSE paths arise similarly to what was found for the KE? In other terms, what is the role played by nonlinear intrinsic mechanisms in the GS/GSE low-frequency variability?

To investigate the issue of the degree of dynamic similarity between GSE and KE and, more in general, to further assess the importance of intrinsic oceanic mechanisms produced by barotropic instability processes in the overall lowfrequency variability of the GSE, in this study the reducedgravity shallow water model of Pierini (2006) is implemented (Sect. 2) in a schematic North Atlantic basin that includes essential geometric features of the coast and a realistic zonal width of the integration domain at all latitudes. Moreover, the time-independent climatological wind stress forcing obtained from 41 years of European Centre of Medium-range Whether Forecasts (ECMWF) monthly fields is discussed. In Sect. 3, the simulated GS mean jet and its variability are both presented and compared with satellite altimeter observations; a preliminary analysis of the intrinsic low-frequency variability of the GS system in terms of dynamical systems theory is also presented. Conclusions are finally drawn in Sect. 4.

\section{The reduced-gravity shallow water model and wind forcing}

As shown by Dengg et al. (1996), the GS system is subdivided into the Loop Current located in the Gulf of Mexico, the Florida Current, the GS proper, the GSE and the continuation of the jet into the North Atlantic and Azores Currents. The GS starts flowing into the northwest Atlantic Ocean from the Florida Straits, and then follows the Florida coasts as a stable parallel flow (the Florida Current). The GSE then forms after separation of the GS past Cape Hatteras and invades a large part of the northern North Atlantic. To possibly capture these features, the domain of integration is chosen so that the large-scale shape of the continental boundaries, between the latitudes $60^{\circ} \mathrm{N}$ and $10^{\circ} \mathrm{S}$, is included. Moreover, a Southern Hemisphere belt delimited by the latitude $\phi=10^{\circ} \mathrm{S}$ allows one to take into account aspects of the equatorial ocean dynamics.

Pierini (2008) showed that elements of realism in the geometry of the domain of integration can play a crucial role in shaping the low-frequency variability of the KE, so that in a process-oriented approach, a correct latitudinal extension (that insures a correct vorticity input in the oceanic interior) and a relatively realistic schematic coastline (on the western side of the ocean in particular) are essential. For the latter, the removal of small-scale coastal features allows one to avoid undesirable instabilities related to the limited resolution, without affecting the basic large-scale features of the flow. Following this approach we have defined a coastline (Fig. 1) composed of a number of connected lines defined on the $\mathrm{x}-\mathrm{y}$ plane of a Cartesian coordinate system by means of a sinusoidal projection. The zonal width extends between the longitudes $75^{\circ} \mathrm{W}$ and $15^{\circ} \mathrm{E}$, spanning the entire North Atlantic basin. Several segments connect eleven points along the western boundary: $\mathrm{A}=\left(10^{\circ} \mathrm{S}\right.$; $\left.34^{\circ} \mathrm{W}\right), \mathrm{B}=\left(6^{\circ} \mathrm{S} ; 35^{\circ} \mathrm{W}\right), \mathrm{C}=\left(9^{\circ} \mathrm{N} ; 60^{\circ} \mathrm{W}\right), \mathrm{D}=\left(16^{\circ} \mathrm{N}\right.$; $\left.61^{\circ} \mathrm{W}\right), \mathrm{E}=\left(22^{\circ} \mathrm{N} ; 79^{\circ} \mathrm{W}\right), \mathrm{F}=\left(31^{\circ} \mathrm{N} ; 81^{\circ} \mathrm{W}\right), \mathrm{G}=\left(35^{\circ} \mathrm{N}\right.$; $\left.75^{\circ} \mathrm{W}\right), \mathrm{H}=\left(40^{\circ} \mathrm{N} ; 73^{\circ} \mathrm{W}\right), \mathrm{I}=\left(46^{\circ} \mathrm{N} ; 52^{\circ} \mathrm{W}\right), \mathrm{J}=\left(54^{\circ} \mathrm{N}\right.$; $\left.56^{\circ} \mathrm{W}\right)$ and $\mathrm{K}=\left(60^{\circ} \mathrm{N} ; 63^{\circ} \mathrm{W}\right)$. The eastern boundary of the Atlantic basin is delimited by a border that excludes the adjacent seas from the integration domain, and the western coasts of Africa are represented by a circle. The use of this schematic coastline carries the advantages of filtering out small-scale motion induced by analogues of small-scale features of the coastlines (irrelevant when analyzing large-scale flows), and of highlighting the role played by the coastline in shaping the ocean dynamics. 


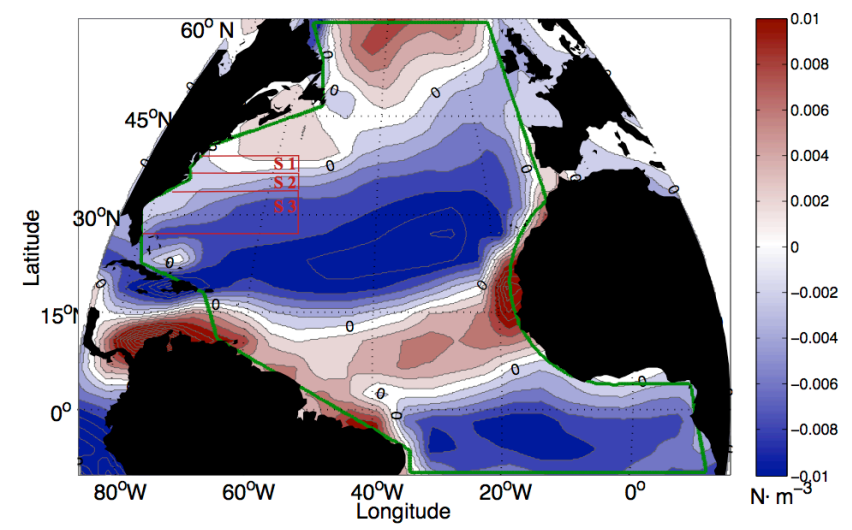

Fig. 1. Domain of model integration (delimited by the green curve) and climatological surface wind-stress curl obtained from 41 years (1961-2001) of ECMWF wind-velocity fields retrieved from the ERA-40 re-analysis project. S1, S2, and S3 indicate sectors in which the flow kinetic energy is integrated to obtain the time series shown in Figs. 4 and 9.

The flow in this model is governed by the reduced-gravity, nonlinear shallow water equations:

$$
\begin{aligned}
\frac{\partial \boldsymbol{u}}{\partial t}+(\boldsymbol{u} \cdot \nabla) \boldsymbol{u}+f \boldsymbol{k} \times \boldsymbol{u}= & -g^{\prime} \nabla \tilde{\eta}+\frac{\boldsymbol{\tau}}{(\rho H)} \\
& +K_{H} \nabla^{2} \boldsymbol{u}-\gamma \boldsymbol{u}|\boldsymbol{u}| \\
\frac{\partial \tilde{\eta}}{\partial t}+\nabla \cdot(H \boldsymbol{u})=0 &
\end{aligned}
$$

where $\boldsymbol{k}=(0,0,1), \boldsymbol{u}=(u, v, 0)$ is the vertically averaged horizontal velocity, $f=2 \Omega_{\text {earth }} \sin \varphi$ the vertical component of the planetary vorticity (where $\Omega_{\text {earth }}=7.3 \times 10^{-5} \mathrm{rad} \mathrm{s}^{-1}$ is the rotation rate of the earth and $\varphi$ is the local latitude), $g^{\prime}=g\left(\rho_{2}-\rho_{1}\right) / \rho$ the reduced gravity (where $g$ is the acceleration of gravity, $\rho_{1}$ and $\rho_{2}$ are the constant upper and lower layer densities, respectively, and $\rho$ is a mean density), $\tilde{\eta}$ is the thermocline displacement (positive downward), $H=D+\tilde{\eta}$ the upper (active) layer thickness (where $D$ is the undisturbed layer thickness), $\boldsymbol{\tau}=\left(\tau_{\mathrm{x}}, \tau_{\mathrm{y}}, 0\right)$ the surface wind stress, $K_{H}$ the lateral eddy viscosity coefficient, and $\gamma$ the quadratic interfacial friction coefficient. No-slip boundary conditions are applied along all lateral walls.

Although in the context of the GS system the applicability of such a simplified model may appear inappropriate (e.g. because baroclinic instability, the barotropic component and at least a second active layer that can include a deep current are all absent in the dynamics), Eqs. (1) do account for the formation of meanders and of intrinsic low-frequency fluctuations generated by barotropic instability, which are found to be essential in the KE case (Pierini, 2006; Pierini et al., 2009). Since the equations are solved numerically through an explicit finite-difference method (Pierini, 2006), the use of a model without the external mode allows for a substantial reduction of the integration time. This is particularly useful when many simulations are required for the analysis of
Table 1. Parameter values used in the simulations.

\begin{tabular}{ll}
\hline Parameter & Value \\
\hline Depth & $D=1000 \mathrm{~m}$ \\
Grid size & $\Delta x=\Delta y=20 \mathrm{~km}$ \\
Time step & $\Delta t=20 \mathrm{~min}$ \\
Reduced-gravity & $g^{\prime}=0.021 \mathrm{~m} \mathrm{~s}^{-2}$ \\
Interfacial friction & $\gamma=5 \times 10^{-4} \mathrm{~m}^{-1}$ \\
Eddy viscosity coefficient & $K_{H}=100 \mathrm{~m}^{2} \mathrm{~s}^{-1}$ \\
\hline
\end{tabular}

flow behaviour in the framework of dynamical systems theory (Sect. 3.3).

The parameter values used are listed in Table 1. The grid size of $20 \mathrm{~km}$ makes this model eddy-permitting, and Chassignet and Marshall (2008) recognized that this resolution is sufficient to reproduce the main features of the GS. The time step of $20 \mathrm{~min}$ is required by the Courant-FriedrichsLewy condition for numerical stability. The value of $g$ ' was derived from mean densities obtained from vertical sections of the WOCE Atlas (http://sam.ucsd.edu/). The time integration is carried out starting from motionless initial conditions for a time interval of $50 \mathrm{yr}$. Several values of the lateral eddy viscosity $K_{H}$ are considered in sensitivity experiments (Sect. 3.3), but the reference value $K_{H}=100 \mathrm{~m}^{2} \mathrm{~s}^{-1}$ is chosen for the basic numerical experiments described in Sects. 3.1 and 3.2. Finally, the sea surface height (SSH) $\eta$ (positive upward) is computed from $\tilde{\eta}$ through the formula $\eta \cong \tilde{\eta}\left(\rho_{2}-\rho_{1}\right) / \rho$.

The degree of realism introduced by the schematic coastline requires an analogous degree of realism in the wind forcing. Thus, zonal and meridional components of the wind velocity, provided by the ECMWF, have been retrieved from the ERA-40 re-analysis project (Uppala et al., 2005) and interpolated onto the domain of integration. Forty-one years (1961-2001) of monthly mean fields have been used to calculate a climatological surface wind velocity with a spatial resolution of $4^{\circ}$ in both horizontal directions; the corresponding surface wind stress field was then computed through classical bulk formulae. The obtained steady surface wind stress climatology is finally used to force the model: the corresponding wind stress curl is shown in Fig. 1. The choice of neglecting all sort of variability in the forcing (from the short-term to the seasonal and low-frequency time scales) is a necessary element when investigating fluctuations of intrinsic origin (e.g. Dijkstra, 2005).

\section{Numerical results}

\subsection{The climatological flow}

In this section an analysis of the time-averaged model solution and relative comparison with altimeter data is presented 

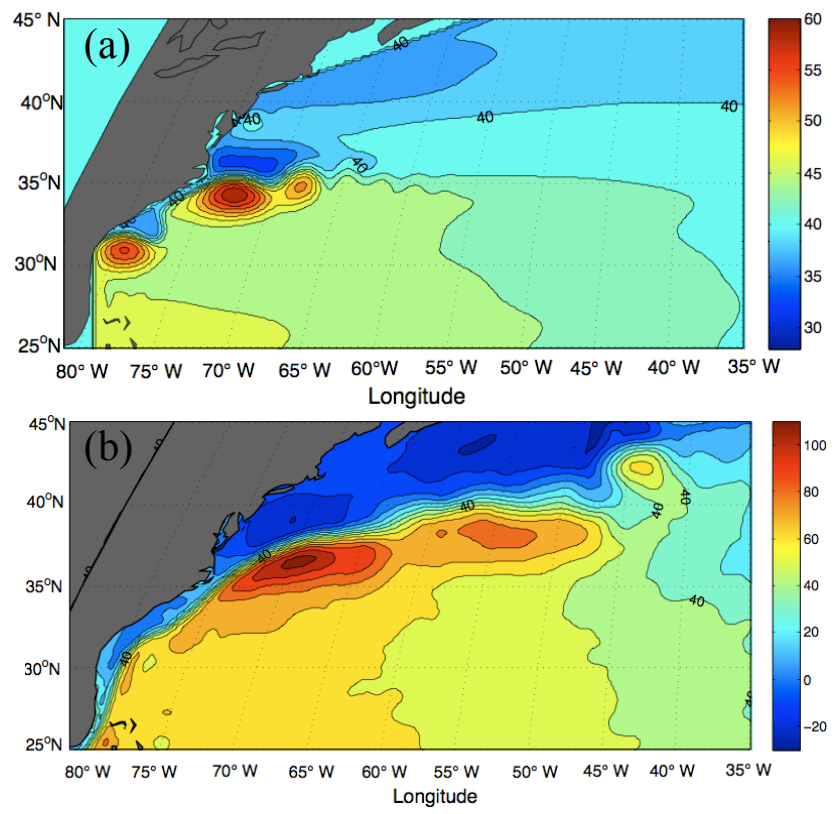

Fig. 2. (a) 26-yr time-mean of the simulated SSH (cm). (b) 18yr (1993-2010) climatology of the absolute dynamic topography (distributed by AVISO/Cnes) (cm). A 40-cm offset has been added to the modeled SSH to make the modeled and observed GSE axis comparable.

in order to identify important modelled GS features that are in agreement with observations. After the model spin-up (see the time series in Fig. 4 below) a time average of the SSH over the $26-\mathrm{yr}$ period $t=24-50 \mathrm{yr}$ is computed. The resulting mean SSH field (shown in Fig. 2a) can be compared with the 18-yr climatology (Fig. 2b) of the absolute dynamic topography (ADT) derived from satellite altimeter products of Salto/Duacs and distributed by Aviso, with the support from Cnes: http://www.aviso.oceanobs.com/duacs/. Since the GSE axis is well represented by the $40-\mathrm{cm}$ contour (see Fig. 2b) and by the 0 -cm contour of the modeled jet, in order to allow for an easier comparison, a 40-cm offset has been added to the simulated SSH (the same change has been applied to all the subsequent figures). To analyse the simulated flow features we divide the GS system into three regions: (i) the Florida Current (FC) region, (ii) the GS and upstream GSE region, and (iii) the downstream GSE region.

(i) The FC is a northward flowing current which follows the Florida coastline: its transport is the result of two main contributions, the Antilles Current and the Loop Current, the latter being dominant. The Loop Current originates in the Gulf of Mexico and moves into the GS through the Florida Straits; estimates provided by Wilson and Johns (1997) suggest a volume flux of $17.5 \mathrm{~Sv}$ through this passage. The GS is highly variable in that location and may in part account for the variability of the FC that is, in turn, affected by both seasonal and interannual changes. The numerical solution (Fig. 2a) yields a weak recirculation cell at its southern edge, including the Antilles Current (which extends along the northern boundary of the Caribbean archipelago up to the Florida coasts). The present model solution does not include the region of the Gulf of Mexico, so the simulated response does not take into account dynamical processes occurring there. This implies that the simulated FC, which correctly flows along the coastline, is weaker than the observed one. Once the FC reaches the South Carolina coasts a seaward deflection of the mean flow is observed (Fig. 2b). Bane and Dewar (1988) noticed that the presence of a topographic irregularity known as the Charleston Bump, off South Carolina $\left(\right.$ near $\left.31^{\circ} \mathrm{N}\right)$, may affect the path of the FC. They also noticed that such a deflection presents a bimodal character, with the FC assuming alternatively a weakly and a strongly deflected state. A much more pronounced seaward deflection of the FC is also present in the model simulation in the correct location, and it is represented, in Fig. 2a, by the northern edge of a strong anticyclonic recirculation gyre centred at $31^{\circ} \mathrm{N}-$ $78^{\circ} \mathrm{W}$ (the latter being also much stronger than the real one). This deflection breaks the meridional orientation of the FC and, since the reduced-gravity model does not take into account topographic effects, the modelled premature separation from the coast must be due to a combination of inertial effects and coastline orientation. Although the mutual interactions between the GS and the topographic irregularity are certainly important effects, we conjecture that the deflection may be partly due also to a premature separation of the GS. Premature separations have also been found in model studies on the role of the viscosity parameterization on the GS separation at Cape Hatteras (Chassignet and Garaffo, 2001) and in Parallel Ocean Climate Model simulations (Schmeits and Dijkstra, 2001): the GS separates either too far south compared to reality or too far north. However, Adcroft and Marshall (1998) demonstrated that piecewise-constant coastlines exert a spurious form stress on model boundary currents that depends on the implementation of lateral boundary conditions; such form stress can, in some cases, introduce premature WBC separation.

(ii) At about $33^{\circ} \mathrm{N}$, beyond the seaward deflection, the modelled GS rejoins the coastline and continues northward toward Cape Hatteras, where, at $35^{\circ} \mathrm{N}$ it separates from the coast near the correct location (Fig. 2b), although with a more zonally oriented path in comparison with the observed flow. In view of the lack of any topographic effect in the adopted reduced-gravity model, this result is in support of the inertial overshooting as a major separation mechanism, whose relevance in the GS separation process was stressed in a recent laboratory study (Pierini et al., 2011). A more quantitative model-data comparison of the flow just after separation can be performed through Fig. 3, where the observed (solid line) and modelled (dashed line) zonal geostrophic velocity sections at $35^{\circ} \mathrm{N}$ are plotted. The dashed line (26-year mean of the model response) shows that the model simulates a strong zonal current whose maximum intensity is confined to the west of the basin: this is also evident in the observed 


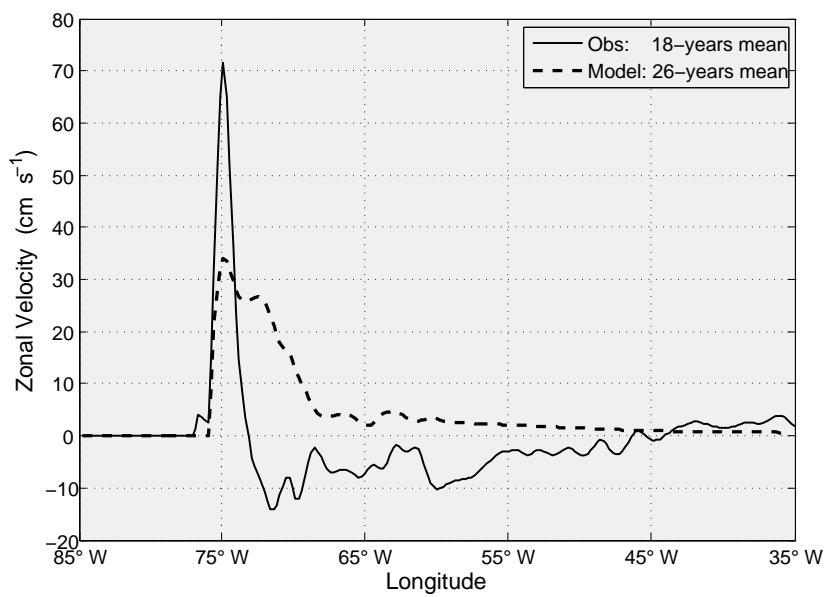

Fig. 3. Zonal geostrophic velocity sections at $35^{\circ} \mathrm{N}$ : $18-\mathrm{yr}$ (19932010) climatology computed from altimeter data distributed by AVISO/Cnes (solid line); 26-yr mean of model response (dashed line).

climatology, and at the same location, but with an amplitude that is about twice the modelled one and with a narrower zonal extension. The upstream GSE is basically a front separating a southern anticyclonic gyre from a northern cyclonic recirculation gyre, whose observed and modelled extensions and locations are in substantial agreement, as shown in Fig. 2a, b (it should be noticed, however, that the modelled anticyclonic gyre is decomposed into two recirculation cells).

(iii) Further downstream, the observed GSE deviates more northward when compared with the modelled flow. Eastward of $\sim 65^{\circ} \mathrm{W}$ the modelled GSE loses its inertial feature and starts broadening (Fig. 2a), in disagreement with the observed flow (Fig. 2b), which yields two further elongated recirculation cells at those eastern locations. Nonetheless, the $40-\mathrm{cm}$ contour lines are in good agreement up to the point where the GSE rejoins gradually the interior circulation of the North Atlantic Ocean.

\subsection{Intrinsic low-frequency variability}

In order to explore the intrinsic GSE low-frequency variability we follow Pierini (2006) in deriving time series (shown in Fig. 4a) of the kinetic energy integrated over the sectors S1, S2, and S3 defined in Fig. 1. Sector S1 includes only partially the jet variability and displays low energy values; in contrast, sector S2 represents most of the variability of the jet, and includes several oscillations superimposed on a higher amplitude relaxation-type oscillation; as for sector S3, since it is far from the jet axis it displays lower amplitude oscillations. In summary, all signals display complex oscillatory behaviour due to intrinsic instabilities, and the corresponding spectra within the more intense region of variability, S1US2, yield peaks at 1.0, 1.2, 3.9, 6.6, and $13.0 \mathrm{yr}$ (Fig. 4b).
In order to analyze the spatial structure of the variability, yearly snapshots (sampled at the first day of each year) of the modelled 40-cm SSH contours have been overlapped in Fig. 5a. Off North Carolina the modelled GS yields a stable separation location at $35^{\circ} \mathrm{N}$, in good agreement with observations (e.g. Lee and Cornillon, 1995). In its upstream region $\left(\lambda \cong 75^{\circ} \mathrm{W}-70^{\circ} \mathrm{W}\right)$ the modelled GSE axis displays two different meridional locations denoted with green and blue contours, respectively (orange contours indicate intermediate locations). The mean meridional position of the green paths is on average at $\sim 35^{\circ} \mathrm{N}$; this state gradually shifts northward reaching the blue paths position at $\sim 36^{\circ} \mathrm{N}$. The analogous paths obtained from altimeter data (Fig. 5b) display a meridional shifting of the jet, whose latitudinal location can be persistent during two or three years. While (as already noticed in the preceding section) the real upstream GSE is more northward oriented compared with the modelled jet, it is interesting to notice that the range of the cross-stream displacement of the modelled jet axis is in good agreement with that of observations. Finally, further east $\left(\lambda \cong 70^{\circ} \mathrm{W}-65^{\circ} \mathrm{W}\right)$, the modelled GSE (Fig. 5a) displays higher amplitude oscillations with a more pronounced meandering activity: all the contours are involved in a latitudinal shift of about $1^{\circ}-2^{\circ}$. In general blue contours follow a slightly more straight path, with relatively small amplitude meandering when compared to the green contours. These stronger oscillations are absent in the real GSE in the same longitudinal band, although a weak meandering activity (absent in the first part of the jet) does occur (Fig. 5b).

The GSE latitudinal oscillation is shown in Fig. 6 through a Hovmöller $(\phi$-t $)$ diagram in which the zonal geostrophic velocity at $75^{\circ} \mathrm{W}$ is plotted as a function of time during the 26-yr interval of our analysis. The strong core of the stream is on average located at $35^{\circ} \mathrm{N}$, but transitions between the high and low latitudinal positions are also evident. The stream is persistent over an interval of about $3-4 \mathrm{yr}$ while transitions occur over shorter periods, generally of 1-2 yr.

Figures 7 and 8 show observed annual averages and model snapshots of the SSH, respectively, and reveal synoptic features of the GS/GSE associated with the states analyzed in Fig. 5. In the first two panels of Fig. 7 (years 1995, 2000) the GSE is characterized by a deflection of the jet axis at $\sim 70^{\circ} \mathrm{W}$ (where it reaches the latitude $\sim 38^{\circ} \mathrm{N}$ ) from an almost SW-NE orientation to the west to an almost zonal orientation to the east, accompanied by a weak meandering further east: this state corresponds to the blue curves of Fig. 5b. On the other hand, in the third and fourth panels (years 1998, 2006), at $\sim 70^{\circ} \mathrm{W}$ the axis reaches only the latitude $\sim 36^{\circ} \mathrm{N}$, moreover the SW-NE orientation extends up to $66^{\circ}-67^{\circ} \mathrm{W}$ followed by a pronounced meandering activity further east: this state corresponds to the green curves of Fig. 5b. This behaviour is similar to that of the $\mathrm{KE}$, for which a more northerly mean path is associated with a persistent meandering state, whereas a more southerly mean path is characterized by a more convoluted and variable jet (e.g. see Figs. 2 

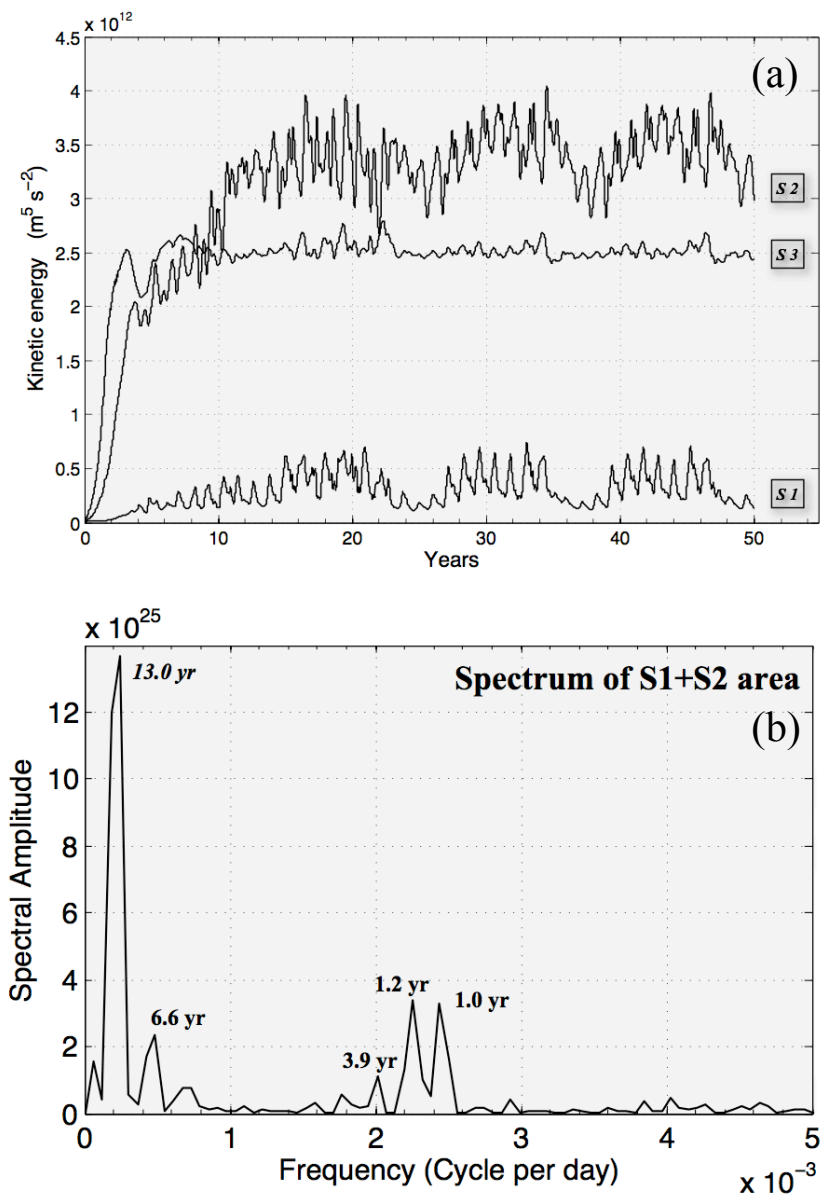

Fig. 4. (a) Time series of the kinetic energy integrated over sectors S1, S2 and S3 (see Fig. 1). (b) Spectral amplitude of the kinetic energy integrated over the sector S1US2.

and 3 of Qiu and Chen, 2010; see also Fig. 2 of Pierini et al., 2009, for a model-data comparison). However, the changes in both the latitudinal location of the jet and the downstream meandering activity are clearly much weaker in the GSE. Finally, despite the differences between modelled and observed patterns already noticed in Fig. 2, in the panels of Fig. 8 some analogies with the above mentioned states can be recognized: in the first two panels ( $t=26,15 \mathrm{yr}$ ) the upstream GSE yields a more northern location and a weaker meandering downstream than in the other two panels $(t=8,9 \mathrm{yr})$.

\subsection{Preliminary analysis of the variability in terms of dynamical systems theory}

The origin of the GSE low-frequency variability can also be analyzed in the framework of dynamical systems theory by studying the transition behaviour of the modelled flows when a control parameter is varied, thus obtaining valuable insights into the role played by such a parameter in shaping the variability (Dijkstra, 2005). Carrying out a thorough analysis of that kind in a problem like the present one with $\mathrm{O}\left(10^{6}\right)$ degrees of freedom can only be done by performing a large number of forward time integrations: this in turn would allow one to obtain an empirical bifurcation diagram and various statistical measures of flow behaviour (e.g. as done by Pierini et al., 2009, for the KE case). A study of this kind is beyond the scope of this paper; nonetheless, in this section we present a brief preliminary analysis in terms of dynamical systems theory by varying the dissipative parameter $K_{H}$.

Figure 9a shows the time series of kinetic energy integrated over the GSE area (sector S1US2 $\cup S 3$ of Fig. 1) obtained by varying $K_{H}$. The time series with the highest kinetic energy (curve labelled 1) represents the reference simulation analyzed in the previous sections. Figure $9 \mathrm{~b}$ shows orbits projected onto the $\mathrm{KINE}(\mathrm{S} 1)-\mathrm{KINE}(\mathrm{S} 2 \cup \mathrm{S} 3)$ plane for the different values of $K_{H}$. Within the range $K_{H}=100$ $300 \mathrm{~m}^{2} \mathrm{~s}^{-1}$ several attractors emerge: a stationary state, small amplitude oscillations, higher energy periodic oscillations, and a large amplitude composite oscillation. The red points in Fig. 9b identify the steady states of the system while periodic oscillations are shown as closed curves. In a first small amplitude range (range "A" in Fig. 9a) one can identify a first Hopf bifurcation leading to small amplitude periodic oscillations.

The green curve $\left(K_{H}=200 \mathrm{~m}^{2} \mathrm{~s}^{-1}\right)$, that after spinup leads again to a steady state, marks the transition to a higher amplitude range (range "B"). A new Hopf bifurcation arises between curves 4 and $3\left(K_{H}=160,140 \mathrm{~m}^{2} \mathrm{~s}^{-1}\right)$. For $K_{H}=$ $140,120 \mathrm{~m}^{2} \mathrm{~s}^{-1}$ the two periodic oscillations are visible as limit cycles in Fig. 9b; the amplitude of the oscillation approximately doubles for $K_{H}=120 \mathrm{~m}^{2} \mathrm{~s}^{-1}$. Moreover, inspection of Fig. 9a, b suggests the existence, in range B, of a global bifurcation between curves 2 and 1 marking the transition from small amplitude, nearly monochromatic oscillations to the large amplitude relaxation-type oscillation (curve 1) discussed in the preceding sections.

\section{Summary, discussion and conclusions}

In this paper it was shown that a reduced-gravity (therefore without baroclinic instability, topographic effects and buoyancy forcing) nonlinear primitive equation ocean model, forced by a steady climatological wind field derived from ECMWF data and implemented with schematic but reasonably realistic coastlines, produces a mean flow and an intrinsic low-frequency variability of the GS/GSE system that is, in some of its prominent features, in reasonable agreement with observations. More specifically:

- for the climatological flow significant agreement is found for the location of the FC seaward deflection and corresponding southern recirculation gyre, for the location of the GS separation, and for the location and extension of the upstream GSE northern and southern recirculation gyres; 

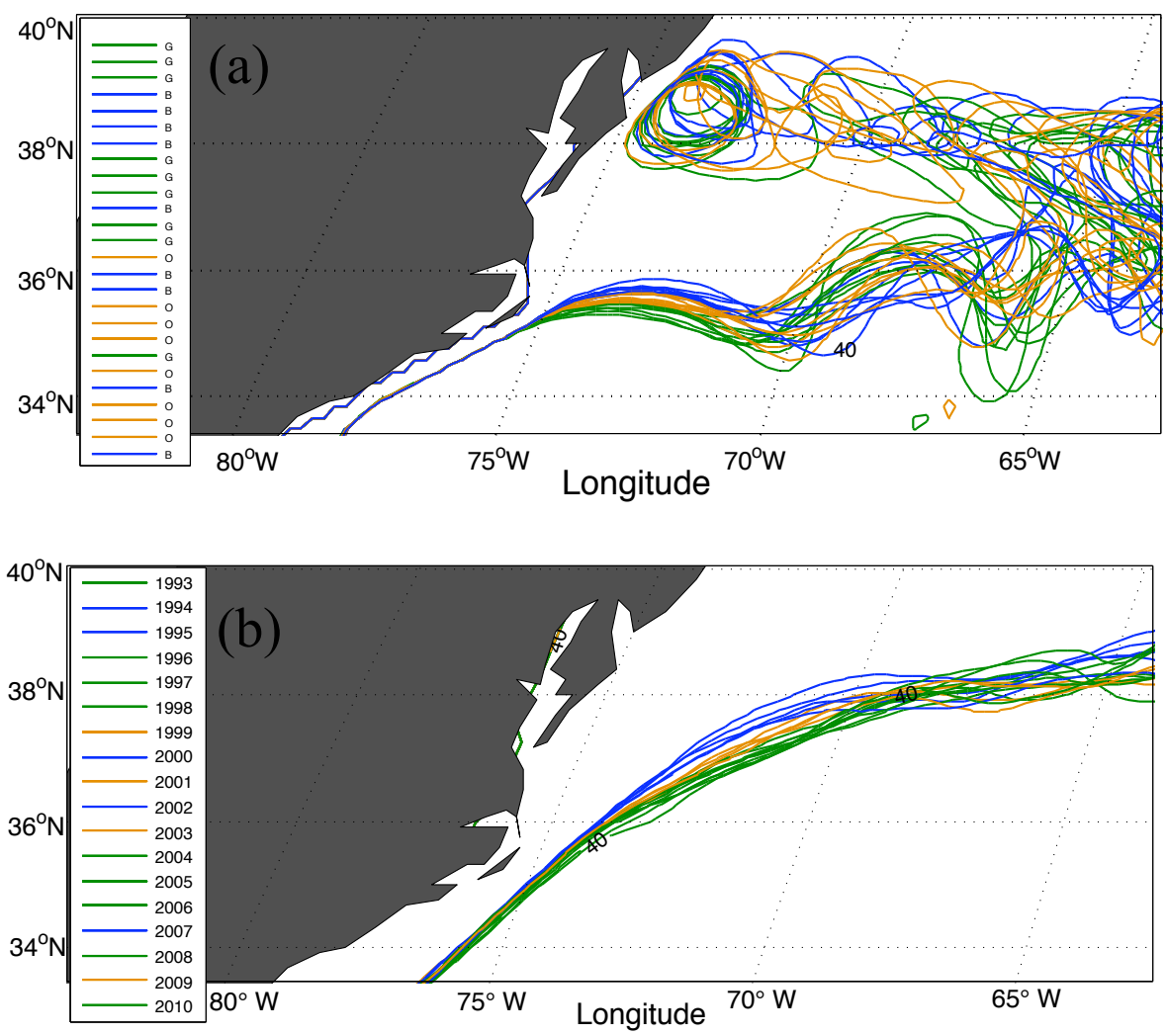

Fig. 5. (a) Paths of the modelled GS and GSE defined by the $40-\mathrm{cm} \mathrm{SSH}$ contours (the 26 curves represent the last 26 years of the integration). The blue (B) and green (G) contours show the higher and lower latitudinal locations of the jet, respectively; the orange contours (O) correspond to intermediate locations (the legend shows the temporal sequence of the contours). (b) Paths of the GS and GSE defined by the 40-cm observed absolute dynamic topography (distribuited by AVISO/Cnes). The blue and green contours show the higher and lower latitudinal locations of the jet, respectively; the orange contours correspond to intermediate locations (the legend refers to the corresponding annual average).

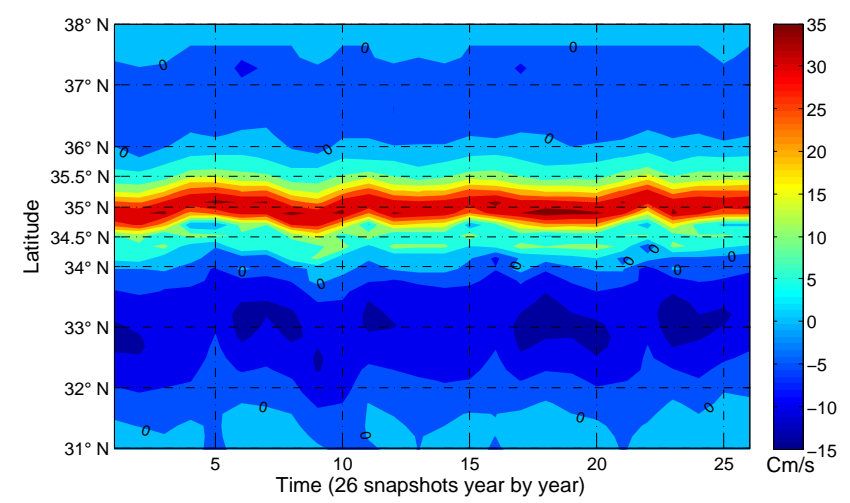

Fig. 6. Contour plot of the simulated zonal geostrophic velocity at $75^{\circ} \mathrm{W}$ as a function of time.

- for the intrinsic low-frequency variability significant agreement is found for the existence of two main GSE axis shapes and locations in the band $\lambda \cong 75^{\circ} \mathrm{W}-65^{\circ} \mathrm{W}$ (that identify two preferred states of the jet), for the range of the cross-stream displacement of the modelled jet axis in the same band, and for the occurrence of a meandering activity in the band $\lambda \cong 70^{\circ} \mathrm{W}-65^{\circ} \mathrm{W}$.

A preliminary analysis of the transition behaviour of the flow has also been carried out by varying the lateral eddy viscosity coefficient: relaxation-type oscillations are found to emerge from a global bifurcation, similarly to what was found for the KE within a similar model approach.

It should also be stressed that several modelled features of the GS/GSE mean flow and variability are not in agreement with observations. More specifically:

- for the climatological flow no agreement with observations is found for the amplitude of the modelled FC seaward deflection (which is much stronger than the observed stream), for the modelled upstream GSE location (which is more zonally oriented than the observed jet, and with half the observed current speed), and for the modelled downstream GSE (which is much weaker than the observed jet); 

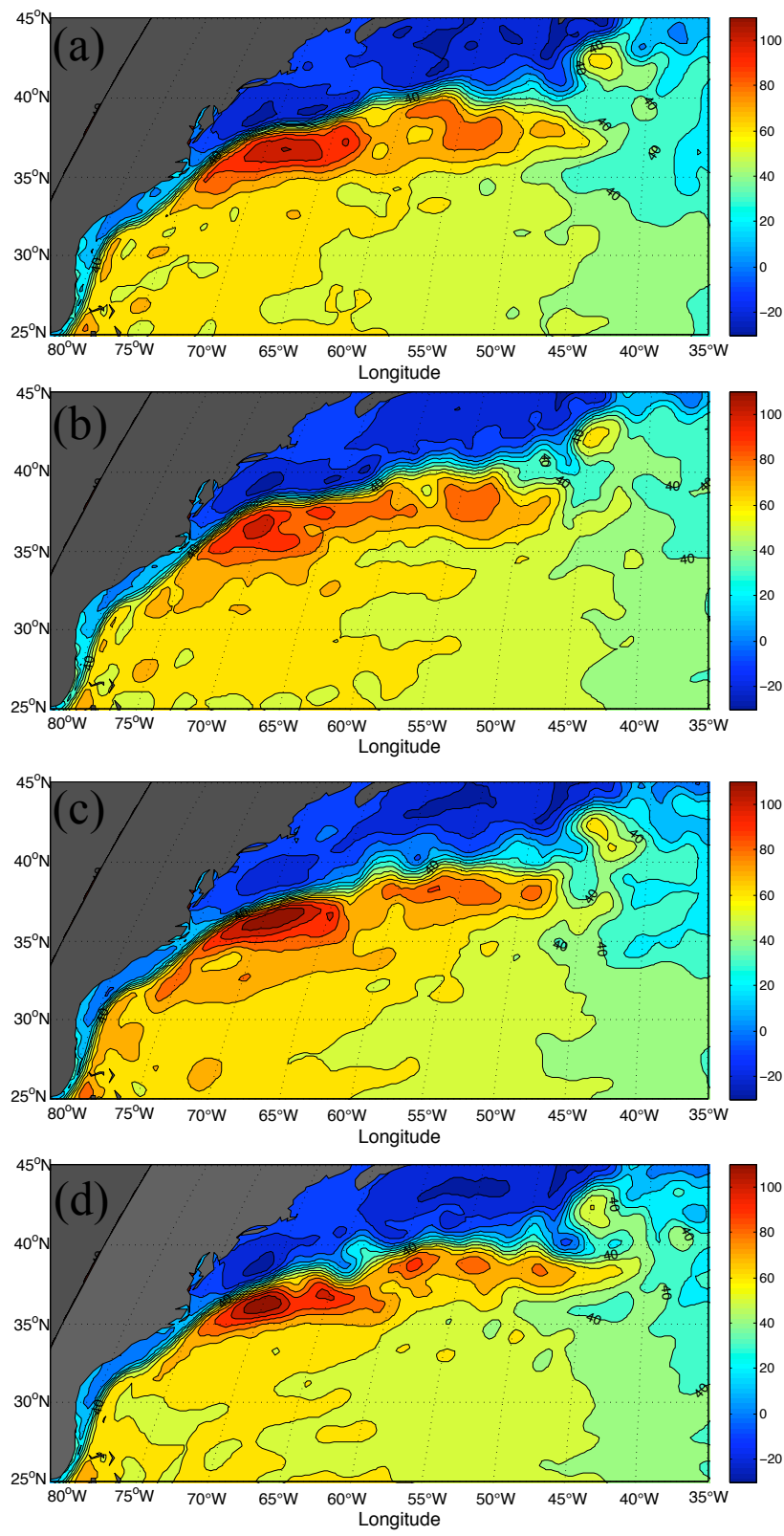

Fig. 7. Annual averages of the absolute dynamic topography distributed by Aviso/Cnes (cm). From top to bottom: year 1995, 2000, 1998, 2006.

- for the intrinsic low-frequency variability no agreement with observations is found for the amplitude of the modelled GSE meandering activity in the band $\lambda \cong$ $70^{\circ} \mathrm{W}-65^{\circ} \mathrm{W}$ (which is much stronger than that of the observed jet).

However, this lack of agreement should not be seen as a drawback of this idealized study, whose aim is only to identify, in a relatively idealized context, the main features present in the observations that are possibly generated by nonlinear intrinsic mechanisms produced by barotropic in-
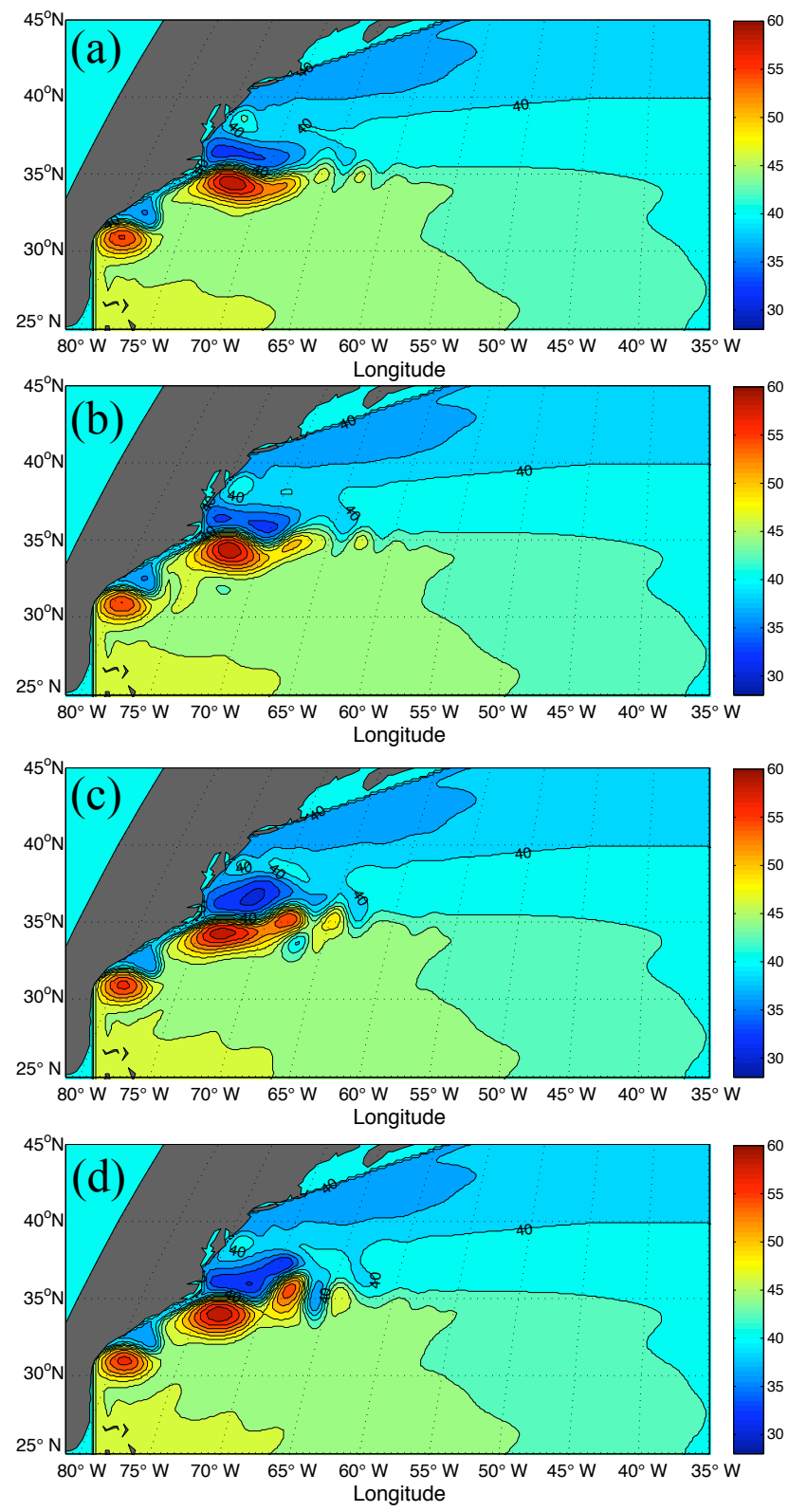

Fig. 8. Simulated snapshots of the SSH $(\mathrm{cm})$ corresponding (from top to bottom) to the first field of $t=26,15,8,9 \mathrm{yr}$.

stability processes. In connection to this, an important point concerns the observed relationship between characteristics of the GSE interannual variability (namely, the meridional shift of the GSE axis) and wind fluctuations associated with the North Atlantic Oscillation (e.g. Frankignoul et al., 2001; Sasaki and Schneider, 2011a). One might argue that since those features are wind-driven they cannot be due to intrinsic effects because, if the latter are the cause, then no direct relationship between GSE changes and wind changes should be expected. 

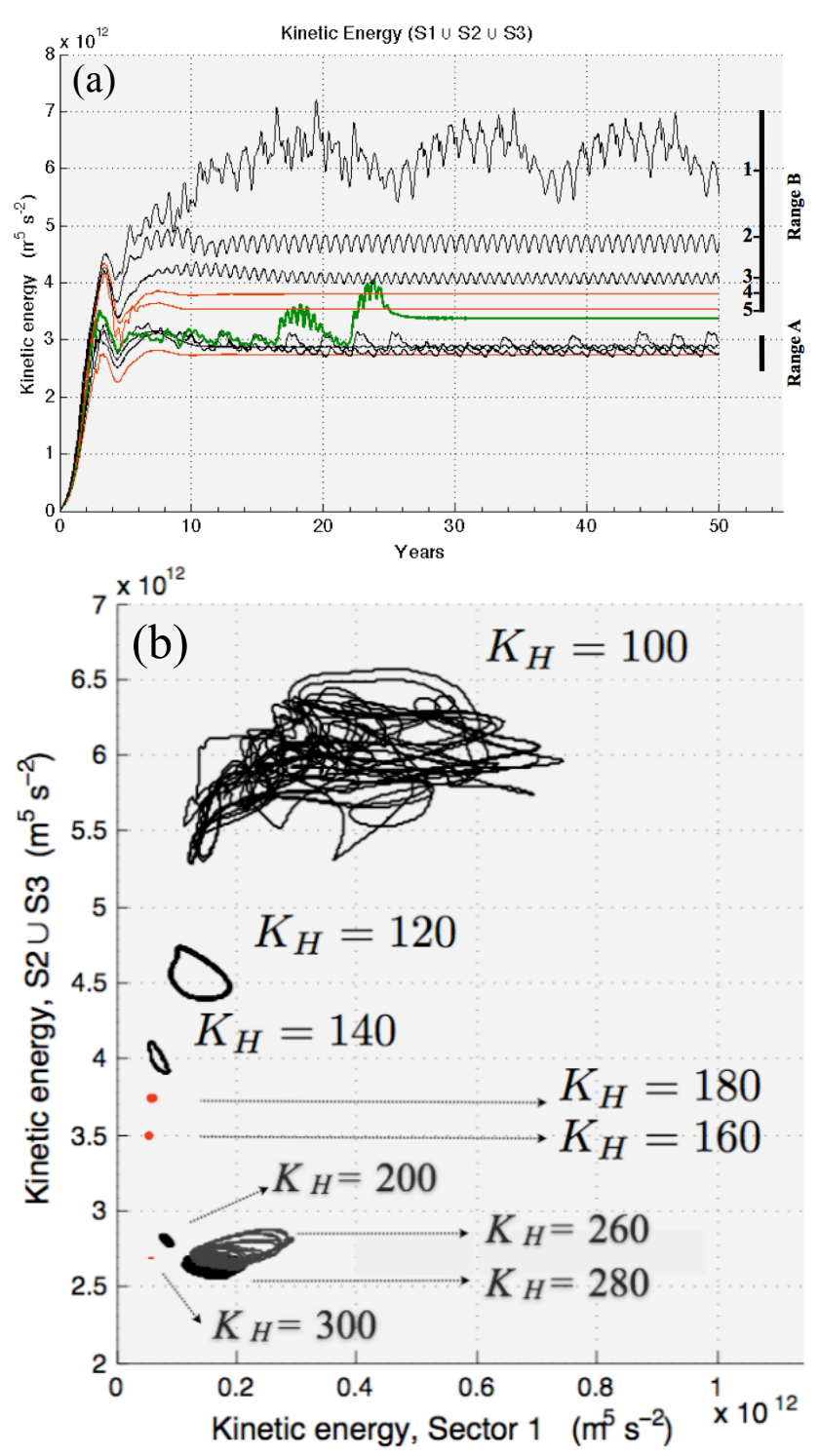

Fig. 9. (a) Time series of the kinetic energy integrated in sec-

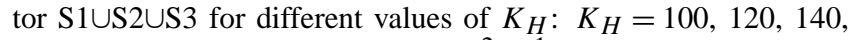
$160,180,200,220,240,260,300 \mathrm{~m}^{2} \mathrm{~s}^{-1}$, corresponding to lines $1, \ldots, 10$, respectively. Two different energetic regimes (Range A and Range B) can be identified; the green line $\left(K_{H}=200 \mathrm{~m}^{2} \mathrm{~s}^{-1}\right)$ marks the transition between them. The red lines identify steady states of the system. (b) Orbits projected onto the (kinetic energy)(S1) - (kinetic energy) (S2 $\cup \mathrm{S} 3)$ plane for different values of $K_{H}$.

However, this simplistic scenario holds only in an autonomous dynamical systems framework. In fact, from a more realistic non-autonomous dynamical systems viewpoint (in which, for example, the system is forced by a mean wind plus a wind noise, and even periodic components) the same relaxation oscillations found with steady forcing can either be triggered by wind variations through the coherence resonance mechanism (e.g. Pierini, 2010, 2011 - section 4a,
2012) or be synchronized (e.g. Anishchenko et al., 2007) or be in weak phase relation with a periodic forcing component (e.g. Pierini, 2011 - section 4b). This suggests that the intrinsic low-frequency variability obtained in a model with steady forcing (such as the present one) can well be correlated with wind variations, as appears from GSE observations. In the companion case of the KE decadal relaxation oscillations, a hypothesis put forward is that the observed Rossby wave signal (found to be correlated with wind stress changes associated with the Pacific Decadal Oscillation) controls the intrinsic KE bimodal variability through a spatial reorganization of the KE flow, from a meridionally broad to a frontal scale structure, thanks to the internal variability of the ocean (Taguchi et al., 2007; Pierini and Dijkstra, 2009). A similar mechanism can be conjectured for the GSE variability as well. Obviously, further modeling studies are needed to support this hypothesis.

Acknowledgements. HD thanks the organizers of the DAMES'2010 conference held on 22-24 September 2010 in Lisbon for the very interesting meeting.

Edited by: R. Donner

Reviewed by: two anonymous referees

\section{References}

Adcroft, A. and Marshall, D.: How slippery are piecewise-constant coastlines in numerical ocean models?, Tellus, 50A, 95-108, 1998.

Anishchenko, V. S., Astakhov, V., Neiman, A., Vadivasova, T., and Schimansky-Geier, L.: Nonlinear dynamics of chaotic and stochastic systems, Springer, 446 pp., 2007.

Auer, S. J.: Five-year climatological survey of the Gulf Stream system and its associated rings, J. Geophys. Res., 92, 11709-11726, 1987.

Bane, J. M. and Dewar, W. K.: Gulf Stream bimodality and variability downstream of the Charleston Bump, J. Geophys. Res., 93, 6695-6710, 1988.

Chassignet, E. P. and Garaffo, Z. D.: Viscosity parameterization and the Gulf Stream separation, in: From Stirring to Mixing in a Stratified Ocean, Proceedings of the 12th 'Aha Huliko' a Hawaiian Winter Workshop, Univ. of Hawaii, edited by: Muller, P. and Henderson, D., 37-41, 2001.

Chassignet, E. P. and Marshall, D. P.: Gulf Stream separation in numerical ocean models, in: Eddy-Resolving Ocean Modeling, edited by: Hecht, M. and Hasumi, H., AGU Monograph Series, pp. 39-62, 2008.

de Coëtlogon, G., Frankignoul, C., Bentsen, M., Delon, C., Haak, H., Masina, S., and Pardaens, A.: Gulf Stream variability in five oceanic general circulation models, J. Phys. Oceanogr., 36, 2119-2135, 2006.

Dengg, J., Beckmann, A., and Gerdes, R.: The Gulf Stream separation problem, in: The Warmwatersphere of the North Atlantic, edited by: Krauss, W., Gebruder Borntrager, pp. 253-290, 1996.

Dijkstra, H. A.: Nonlinear Physical Oceanography: A Dynamical Systems Approach to the Large Scale Ocean Circulation and El Niño, Springer, 532 pp., 2005. 
Dijkstra, H. A. and Ghil, M.: Low-frequency variability of the large-scale ocean circulation: A dynamical systems approach, Rev. Geophys., 43, RG3002, doi:10.1029/2002RG000122, 2005.

Dijkstra, H. A. and Katsman, C.: Temporal variability of the quasigeostrophic wind-driven ocean circulation, Geophys. Astrophys. Fluid Dyn., 85, 195-232, 1997.

Dijkstra, H. A. and Molemaker, M. J.: Imperfections of the NorthAtlantic wind-driven ocean circulation: continental geometry and wind stress shape, J. Mar. Res., 57, 1-28, 1999.

Feliks, Y., Ghil, M., and Robertson, A. W.: The atmospheric circulation over the North Atlantic as induced by the SST field, J. Climate, 24, 522-542, 2011.

Frankignoul, C., de Coëtlogon, G., Joyce, T. M., and Dong, S.: Gulf Stream variability and ocean-atmosphere interactions, J. Phys. Oceanogr., 31, 3516-3529, 2001.

Jiang, S., Jin, F., and Ghil, M.: Multiple equilibria, periodic and aperiodic solutions in a wind-driven, double-gyre, shallow-water model, J. Phys. Oceanogr., 25, 764-786, 1995.

Joyce, T. M., Kwon, Y. O., and Yu, L.: On the relationship between synoptic wintertime atmospheric variability and path shifts in the Gulf Stream and the Kuroshio Extension, J. Climate, 22, $3177-$ 3191, 2009.

Kelly, K. A., Small, R. J., Samelson, R. M., Qiu, B., Joyce, T. M., Kwon, Y., and Cronin, M. F.: Western boundary currents and frontal air-sea interaction: Gulf Stream and Kuroshio Extension, J. Climate, 23, 5644-5667, 2010.

Lee, T. and Cornillon, P.: Temporal variation of meandering intensity and domain-wide lateral oscillations of the Gulf Stream, J. Geophys. Res., 100, 13603-13613, 1995.

McCalpin, J. D. and Haidvogel, D. B.: Phenomenology of the low-frequency variability in a reduced-gravity, quasigeostrophic double-gyre model, J. Phys. Oceanogr., 26, 739-752, 1996.

Pierini, S.: A Kuroshio Extension system model study: decadal chaotic self-sustained oscillations, J. Phys. Oceanogr., 36, 16051625, 2006.

Pierini, S.: On the crucial role of basin geometry in double-gyre models of the Kuroshio Extension, J. Phys. Oceanogr., 38, 13271333, 2008.

Pierini, S.: Coherence resonance in a double-gyre model of the Kuroshio Extension, J. Phys. Oceanogr., 40, 238-248, 2010.

Pierini, S.: Low-frequency variability, coherence resonance and phase selection in a low-order model of the wind-driven ocean circulation, J. Phys. Oceanogr., 41, 1585-1604, 2011.

Pierini, S.: Stochastic tipping points in climate dynamics, Phys. Rev. E, 85, 027101, doi:10.1103/PhysRevE.85.027101, 2012.

Pierini, S. and Dijkstra, H. A.: Low-frequency variability of the Kuroshio Extension, Nonlin. Processes Geophys., 16, 665-675, doi:10.5194/npg-16-665-2009, 2009.

Pierini, S., Dijkstra, H. A., and Riccio, A.: A nonlinear theory of the Kuroshio Extension bimodality, J. Phys. Oceanogr., 39, 2212 2229, 2009.

Pierini, S., Falco, P., Zambardino, G., McClimans, T. A., and Ellingsen, I.: A laboratory study of nonlinear western boundary currents, with application to the Gulf Stream separation due to inertial overshooting, J. Phys. Oceanogr., 41, 2063-2079, 2011.
Qiu, B.: Interannual variability of the Kuroshio Extension system and its impact on the wintertime SST field, J. Phys. Oceanogr., 30, 1486-1502, 2000.

Qiu, B.: The Kuroshio Extension system: its large-scale variability and role in the midlatitude ocean-atmosphere interaction, J. Oceanogr., 58, 57-75, 2002.

Qiu, B. and Chen, S.: Eddy-mean flow interaction in the decadallymodulating Kuroshio Extension system, Deep-Sea Res. II, 57, 1097-1110, 2010.

Rossby, T., Flagg, C., and Donohue, K.: On the variability of Gulf Stream transport from seasonal to decadal timescales, J. Mar. Res., 68, 503-522, 2010.

Sasaki, Y. N. and Schneider, N.: Interannual to decadal Gulf Stream variability in an eddy-resolving ocean model, Ocean Modell., 39, 209-219, 2011a.

Sasaki, Y. N. and Schneider, N.: Decadal shifts of the Kuroshio Extension jet: Application of thin-jet theory, J. Phys. Oceanogr., 41, 979-993, 2011b.

Schmeits, M. J. and Dijkstra, H. A.: Bimodal behavior of the Kuroshio and the Gulf Stream, J. Phys. Oceanogr., 31, 34353456, 2001.

Simonnet, E., Ghil, M., and Dijkstra, H. A.: Homoclinic bifurcations in the quasi-geostrophic double-gyre circulation, J. Marine Res., 63, 931-956, 2005.

Taft, B. A.: Characteristics of the flow of the Kuroshio south of Japan, in: Kuroshio - Its physical aspects, edited by: Stommel, H. and Yoshida, K., University of Tokyo Press, pp. 165-216, 1972.

Taguchi, B., Xie, S.-P., Schneider, N., Nonaka, M., Sasaki, H., and Sasai, Y.: Decadal variability of the Kuroshio Extension: Observations and an eddy-resolving model hindcast, J. Climate, 20, 2357-2377, 2007.

Uppala, S. M., Kållberg, P. W., Simmons, A. J., Andrae, U., da Costa Bechtold, V., Fiorino, M., Gibson, J. K., Haseler, J., Hernandez, A., Kelly, G. A., Li, X., Onogi, K., Saarinen, S., Sokka, N., Allan, R. P., Andersson, E., Arpe, K., Balmaseda, M. A., Beljaars, A. C. M., van de Berg, L., Bidlot, J., Bormann, N., Caires, S., Chevallier, F., Dethof, A., Dragosavac, M., Fisher, M., Fuentes, M., Hagemann, S., Hólm, E., Hoskins, B. J., Isaksen, L., Janssen, P. A. E. M., Jenne, R., McNally, A. P., Mahfouf, J.-F., Morcrette, J.-J., Rayner, N. A., Saunders, R. W., Simon, P., Sterl, A., Trenberth, K. E., Untch, A., Vasiljevic, D., Viterbo, P., and Woollen, J.: The ERA-40 re-analysis, Q. J. Roy. Meteorol. Soc., 131, 2961-3012, doi:10.1256/qj.04.176, 2005.

Wilson, W. D. and Johns, W. E.: Velocity structure and transport in the Windward Islands Passages, Deep-Sea Res., 44, 487-520, 1997.

Zhang, R. and Vallis, G. K.: Impact of great salinity anomalies on the low-frequency variability of the North Atlantic climate, J. Climate, 19, 470-482, 2006. 